\title{
Calculation of Characteristic Impedance for Stripline Based on Planar Circuit and Lateral Equivalent Network
}

\author{
Kai DING*, Takaharu HIRAOKA, Jui-Pang HSU \\ Department of Electrical-Electronics and Information Engineering, Kanagawa University \\ 3-27-1 Rokkakubashi Kanagawa-ku Yokohama, 221-8686, JAPAN \\ r200670072@kanagawa-u.ac.jp*
}

Abstract -- Stripline is a key component for MIC. Therefore, exact calculation of the characteristic impedance is important for analysis and synthesis of stripline circuit. There are many calculation methods for stripline with zero or finite strip thickness. Exact solution for zero thickness was already given by conformal transformation method, but that for finite thinkness is not. So far, relatively exact solution is given by fringing capacitance method. Here, new and systematic mode analysis method is proposed, which is based on lateral equivalent network and can give more exact characteristic impedance for stripline with any thickness including zero thickness. This method is demonstrated to be valid and useful by good agreement with the exact results for zero thickness.

\section{INTRODUCTION}

Stripline is an important transmission line for MIC system. In order to carry out rigorous analysis and design for stripline circuit, exact characteristic impedance for any strip thickness is strongly needed. There are many numerical calculation methods for stripline with zero and finite strip thickness. Exact analytical solution for zero thickness was already well-known and given by conformal transformation method ${ }^{[1]}$, but that for finite thinkness is not available. So far, relatively exact solution are given by fringing capacitance method ${ }^{[2]}$, which has $1.2 \%$ error. Hence, it is important to develop a new method which can give more exact characteristic impedance for any strip thickness including zero. In this paper, a new and systematic method for the calculation of propagation eigenmode (propagation constant and field distribution of TEM mode) for stripline based on lateral equivalent network ${ }^{[4]}$ will be proposed, which is derived from planar circuit equations ${ }^{[3]}$ and mode matching method, and can give exact field distribution for TEM mode. Then characteristic impedance is defined by TEM voltage and current, which can be calculated by line integral of the electric field and contour integral of magnetic field, respectively. These new calculation method is applied to actual stripline structure, dispersion characteristics, field distribution and characteristic impedance are calculated for various dimension. These calculated results are compared with exact solution of zero thickness or conventional solution with good agreement, which demonstrates that our new method is valid and usefull.

\section{LATERAL EQUIVALENT NETWORK ${ }^{[4]}$}

Cross-section of stripline is divided into three uniform regions and two step discontinuities as shown in Fig.1(a). In order to analyze the stripline with any strip thickness, equivalent network in lateral direction is introduced, which is derived from parallel plate planar circuit equations ${ }^{[3]}$ and dependency of $e^{-j \beta_{/ /} y}$ along waveguide. Parameters of the planar circuit equations are given in table 1 . The equivalent network for uniform region is given by multi-transmission line of TE/TM mode and that for step discontinuity by multi-port ideal transformer with mode conversion current source at both side of the step as shown in Fig.1(b) ${ }^{[4]}$. Therefore whole equivalent network in lateral direction is given by Fig.1(c). Through our calculation, half structure of the stripline is treated because of the symmetry at center. Also metal wall is assumed at outside side-wall to make the model simple.

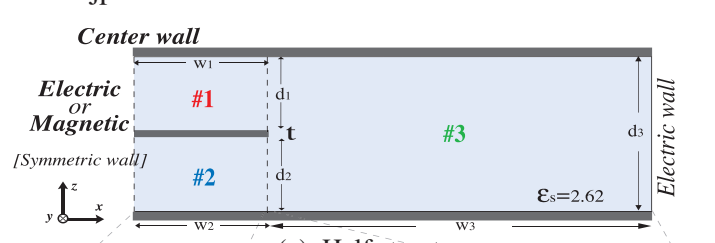

(a) Half structure

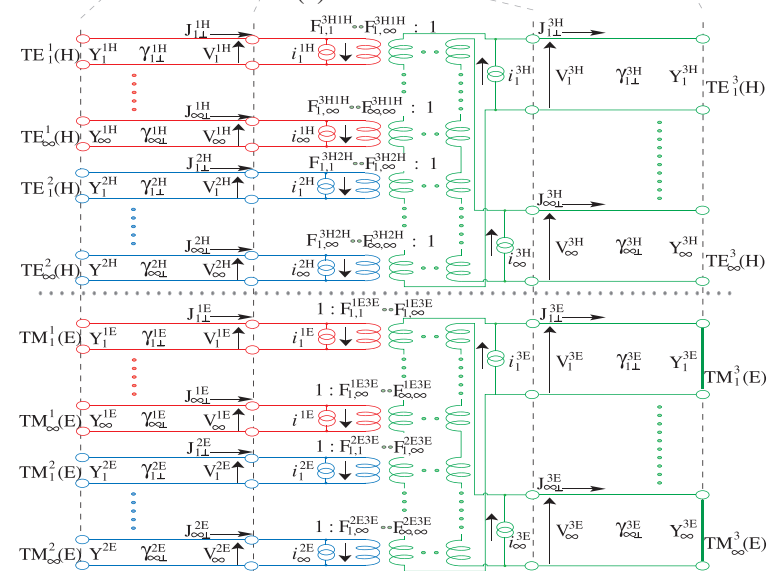

(b) Equivalent network

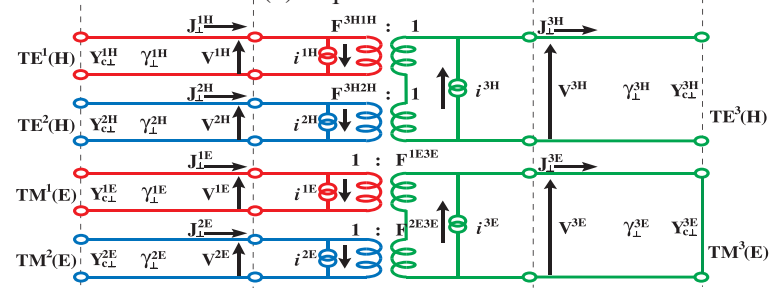

(c) Vector representation

Fig.1 Lateral equavalent network for stripline

\section{CAlculation of PROPAGATION EigENMODE}

For the calculation of propagation eigenmode, eigen-value equation given by eq.(1) is formulated by mode matching condition and lateral equivalent network given by Fig.1.

$$
\left[\begin{array}{ccc}
\overrightarrow{\mathbf{Y}}_{e f f}{ }^{1 E 1 E}+\overline{\mathbf{Y}}_{i n}^{1 E, 2} & \overrightarrow{\mathbf{Y}}_{e f f}{ }^{1 E 2 E} & \overrightarrow{\mathbf{Y}}_{\text {eff }}{ }^{1 E 3 H} \\
\overrightarrow{\mathbf{Y}}_{e f f}{ }^{2 E I E} & \overrightarrow{\mathbf{Y}}_{e f f}^{2 E 2 E}+\overline{\mathbf{Y}}_{i n}^{2 E, 2} & \overrightarrow{\mathbf{Y}}_{e f f}{ }^{2 E 3 H} \\
\overline{\mathbf{Y}}_{e f f}{ }^{3 H 1 E} & \overline{\mathbf{Y}}_{e f f}{ }^{3 H 2 E} & \overline{\mathbf{Y}}_{e f f}{ }^{3 H H}+\overrightarrow{\mathbf{Y}}_{i n}^{3 H, 1}
\end{array}\right]\left[\begin{array}{c}
\mathbf{V}^{1 E, 2} \\
\mathbf{V}^{2 E, 2} \\
\mathbf{V}^{3 H, 1}
\end{array}\right]=0
$$

Propagation constant can be obtained by solving eq.(2).

$$
\operatorname{det}\left(\mathbf{Y}_{\text {eff }}+\mathbf{Y}_{\text {in }}\right)=0
$$

Field distribution of the eigenmode is calculated by eigenvector given by eq.(1). That is, all terminal mode voltage/current are obtained by the eigen-vector and whole equivalent network in lateral direction. Then, any $\mathrm{x}$ dependent mode voltage and mode current distribution is given by eq.(3), where $\mathrm{V}_{\mathrm{p}}^{1,2}$ and $\mathrm{I}_{\mathrm{p}}^{1,2}$ are terminal voltage/current.

$$
\begin{aligned}
& V_{p}(x)=\frac{\sinh \gamma_{p}\left(x-\ell_{2}\right)}{\sinh \gamma_{p}\left(\ell_{1}-\ell_{2}\right)} V_{p}^{1}+\frac{\sinh \gamma_{p}\left(x-\ell_{1}\right)}{\sinh \gamma_{p}\left(\ell_{2}-\ell_{1}\right)} V_{p}^{2} \\
& \vec{I}_{p}(x)=\frac{\sinh \gamma_{p}\left(x-\ell_{2}\right)}{\sinh \gamma_{p}\left(\ell_{1}-\ell_{2}\right)} \vec{I}_{p}^{1}+\frac{\sinh \gamma_{p}\left(x-\ell_{1}\right)}{\sinh \gamma_{p}\left(\ell_{2}-\ell_{1}\right)} \vec{I}_{p}^{2}
\end{aligned}
$$


Hence, field distribusion of the corresponding eigenmode at each uniform region is given by TE/TM mode summation of product of $\mathrm{z}$-dependent mode function in height direction and $\mathrm{x}$ dependent mode voltage and mode current distribution. Final field distribusion is given by eqs.(4) (9).

$$
\begin{aligned}
& E_{z}(x, z)=-\frac{1}{d} \sum_{n} V_{n}^{E}(x) \cdot g_{n}^{E}(z) \\
& H_{z}(x, z)=-\frac{1}{d} \sum_{m} V_{m}^{H}(x) \cdot g_{m}^{H}(z) \\
& E_{y}(x, z)=-\sum_{m} J_{x, m}^{H}(x) \cdot f_{m}^{H}(z)+j \eta_{0} \sum_{n} J_{y, n}^{E}(x) \cdot h_{n}^{E}(z) \\
& H_{y}(x, z)=\sum_{n} J_{x, n}^{E}(x) \cdot f_{n}^{E}(z)+j \frac{1}{\eta_{0}} \sum_{m} J_{y, m}^{H}(x) \cdot h_{m}^{H}(z) \\
& E_{x}(x, z)=\sum_{m} J_{y, m}^{H}(x) \cdot f_{m}^{H}(z)+j \eta_{0} \sum_{n} J_{x, n}^{E}(x) \cdot h_{n}^{E}(z) \\
& H_{x}(x, z)=-\sum_{n} J_{y, n}^{E}(x) \cdot f_{n}^{E}(z)+j \frac{1}{\eta_{0}} \sum_{m} J_{x, m}^{H}(x) \cdot h_{m}^{H}(z)
\end{aligned}
$$

\section{CALCULATION OF CHARACTERISTIC IMPEDANCE}

TEM mode characteristic impedance of stripline, whose structure is shown in Fig. 2 is defined by eq.(10), where TEM voltage $V^{T E M}$ and current $I^{T E M}$ is derived by line integral of electric field $\mathrm{E}_{\mathrm{z}}$ given by eq.(11) and contour integral of magnetic field $\mathbf{H}_{\mathrm{t}}$ given by eq.(12), respectively.

$$
\begin{aligned}
& Z c=\frac{V^{T E M}}{I^{T E M}} \\
& V^{T E M}=\int_{O}^{A} E_{z} d z \\
& I^{T E M}=2 \int_{A B C D} \mathbf{H}_{t} \cdot d \mathbf{s}
\end{aligned}
$$

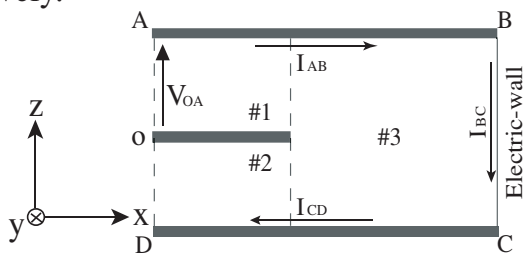

Fig.2 Cross section of half stripline

\begin{tabular}{|c|c|c|}
\hline & z-dependent function & $\mathrm{x}-\mathrm{y}$ dependent function \\
\hline 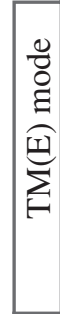 & $\begin{array}{r}f_{n}^{E}(z)=\varepsilon_{s} g_{n}^{E}(z)=\sqrt{\varepsilon_{s}} \sqrt{\varepsilon_{n}} \cos \left(\frac{n \pi}{d} z\right) \\
h_{n}^{E}(z)=-\left(\frac{n \pi}{k_{0} d}\right) \frac{1}{\sqrt{\varepsilon_{s}}} \sqrt{\varepsilon_{n}} \sin \left(\frac{n \pi}{d} z\right) \\
\gamma_{t n}^{E}=\sqrt{\left(\frac{n \pi}{d}\right)^{2}-\varepsilon_{s} k_{0}^{2}}=j \beta_{t n}^{E} \\
\varepsilon_{n}=\left\{\begin{array}{l}1(n=0) \\
2(n \neq 0)\end{array}\right.\end{array}$ & $\begin{array}{c}V_{n}^{E}(x, y)=-E_{z n}^{H}(x, y) \cdot d[V] \\
\mathbf{J}_{n}^{E}(x, y)=\mathbf{H}_{t n}^{H}(x, y) \times \mathbf{k}[A / m] \\
Y_{c n}^{E}=\sqrt{\frac{B_{n}^{E}}{X_{n}^{E}}}=\frac{\omega \varepsilon_{0}}{\beta_{n}^{E}} \frac{1}{d}[S / m] \\
\beta_{t n}^{E}=\sqrt{X_{n}^{E} B_{n}^{E}}[\mathrm{rad} / \mathrm{m}] \\
X_{n}^{E}=\left(\beta_{t n}^{E}\right)^{2} \cdot d / \omega \varepsilon_{0}[\Omega] \\
B_{n}^{E}=\omega \varepsilon_{0} / d\left[S / \mathrm{m}^{L}\right]\end{array}$ \\
\hline 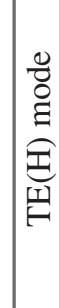 & $\begin{array}{l}f_{m}^{H}(z)=g_{m}^{H}(z)=\sqrt{2} \sin \left(\frac{m \pi}{d} z\right) \\
h_{m}^{H}(z)=\left(\frac{m \pi}{k_{0} d}\right) \sqrt{2} \cos \left(\frac{m \pi}{d} z\right) \\
\gamma_{t m}^{H}=\sqrt{\left(\frac{m \pi}{d}\right)^{2}-\varepsilon_{s} k_{0}^{2}}=j \beta_{t m}^{H}\end{array}$ & $\begin{array}{c}V_{m}^{H}(x, y)=H_{z m}^{H}(x, y) \cdot d[A] \\
\mathbf{J}_{m}^{H}(x, y)=\mathbf{k} \times \mathbf{H}_{t m}^{H}(x, y)[V / m] \\
Y_{c m}^{H}=\sqrt{\frac{B_{m}^{H}}{X_{m}^{H}}}=\frac{\omega \mu_{0}}{\beta_{m}^{H}} \frac{1}{d}[\Omega / m] \\
\beta_{t m}^{H}=\sqrt{X_{m}^{H} B_{m}^{H}}[\mathrm{rad} / \mathrm{m}] \\
X_{m}^{H}=\left(\beta_{t m}^{H}\right)^{2} \cdot d / \omega \mu_{0}[S] \\
B_{m}^{H}=\omega \mu_{0} / d\left[S / m^{L}\right]\end{array}$ \\
\hline
\end{tabular}

Exact electric field $\mathbf{E}_{\mathrm{t}}$ and magnetic field $\mathbf{H}_{\mathrm{t}}$ in the cross-section of stripline can be calculated by solving eigenvalue equation and substituting eigenvector into eq.(4) through eq.(9) as explained in the previous section. Definite integral method by terminal mode voltage and mode current based on lateral equivalent network as shown in Fig. 3 is proposed and explained in the following.

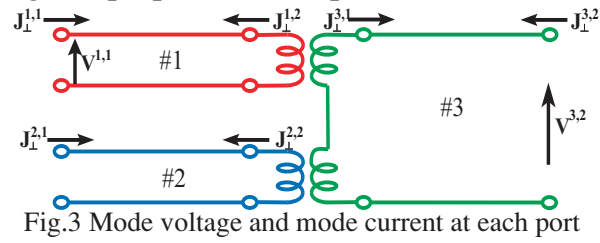

TABLE 1 Relations and parameter for planar circuit
Transverse field $\mathbf{E}_{\mathrm{t}}\left(\mathrm{E}_{\mathrm{x}}, \mathrm{E}_{\mathrm{z}}\right)$ and $\mathbf{H}_{\mathrm{t}}\left(\mathrm{H}_{\mathrm{x}}, \mathrm{H}_{\mathrm{z}}\right)$ are given analytically by eqs.(4),(5),(8) and (9), which make possible to use definite integral for eqs.(11) and (12). Therefore, TEM voltage $V^{T E M}$ is calculated analytically by the following definite integral.

$$
\begin{aligned}
V_{O A}^{T E M} & =-\frac{1}{d} \int_{O}^{A} \sum_{n} V_{n}^{E}(x) \cdot g_{n}^{E}(z) d z \\
& =-\frac{1}{d} \int_{O}^{A} \sum_{n} \frac{\sinh \gamma_{p}\left(x-\ell_{2}\right)}{\sinh \gamma_{p}\left(\ell_{1}-\ell_{2}\right)} V_{p}^{1} \cdot g_{n}^{E}(z) d z
\end{aligned}
$$

Also TEM current given by eq.(12) is calculated analytically by the following definite integral.

$$
\begin{gathered}
I_{A B C D}^{T E M}=2\left(I_{A B}^{T E M}+I_{B C}^{T E M}+I_{C D}^{T E M}\right) \\
I_{A B}^{T E M}=\int_{A}^{B}\left[-\sum_{n} J_{y, n}^{E}(x) \cdot f_{n}^{E}(z)+j \frac{1}{\eta_{0}} \sum_{m} J_{x, m}^{H}(x) \cdot h_{m}^{H}(z)\right] d s \\
I_{B C}^{T E M}=-\frac{1}{d} \int_{B}^{C} \sum_{m}^{H} V_{m}^{H}(x) \cdot g_{m}^{H}(z) d s \\
I_{C D}^{T E M}=\int_{C}^{D}\left[-\sum_{n} J_{y, n}^{E}(x) \cdot f_{n}^{E}(z)+j \frac{1}{\eta_{0}} \sum_{m} J_{x, m}^{H}(x) \cdot h_{m}^{H}(z)\right] d s
\end{gathered}
$$

The accuracy depends only on number of slab-mode considered.

\section{RESUlts of CALCULATION}

So far explained analysis method is applied for practical strip line of various dimension and frequency to prove the validity. A. Convergence behavior of propagation constant

The keypoint of our methed is how many height-mode must be taken into consideration, which is decided by convergence behavior of propagation constant with height-mode. The convergence behavior of TEM and two TE lower modes with heightmode in region \#3 are shown in Fig. 4 for $0.1[\mathrm{~mm}]$.

\section{B. Calculation of Dispersion Characteristics}

Eigenvalue equation given by eq.(1) is solved to give the eigenvalue and eigen-vector for various dimension including zero thickness. Calculated results of lower eigenvalue for zero thickness, which corresponds to propagation constant are shown in Fig. 5 as a function of frequency up to $60[\mathrm{GHz}]$. The calculated propagation constant for TEM mode agrees with exact result of $\beta=\omega \sqrt{\varepsilon \mu}$. Through our calculation $70 \mathrm{TE} / \mathrm{TM}$ slab-modes are taken into consideration. C. Field Distribution for TEM Mode

Field distribution for TEM mode is calculated based on eigen vector, lateral equivalent network and eqs.(4) (9). The calculated results of transverse field distribution ( $\mathrm{x}$ and $\mathrm{z}$ field component) for various dimension are shown in Fig.6. Electric and magnetic field strength $\left(\mathrm{E}_{\mathrm{y}} / \mathrm{H}_{\mathrm{y}}\right)$ of this mode in y direction is calculated to be about $10^{-13}$, which means the mode is TEM.

The electric and magnetic field Ex,Ey,Ez and $\mathrm{Hx}, \mathrm{Hy}, \mathrm{Hz}$ at \#1\#3 and \#2-\#3 step must be continuous because of field theory. Continuity of the $\mathrm{Hx}$ field for $0.1[\mathrm{~mm}]$ is calculated and shown in Fig. 7 with 10 and 80 height-modes in region \#3 considered. D. Investigation of Error for Characteristic Impedance

In order to investigate the accuracy of our method, characteristic impedance of TEM mode for zero thickness are calculated by our method with difinite integral and shown in Fig. 8 with exact results given by conformal transformation ${ }^{[1]}$ and static fringing capacitance approximation ${ }^{[2]}$. It turns out that our present method agree well with exact solution rather than popular static fringing capacitance approximation.

In order to investigate the calculation error in detail, calculation \% error for each method is calculated and shown in Fig.9, which clearly demonstrates that the present method with definte 
integral is better method giving maxmum error of $0.17 \%$. Finally characteristic impedance vs w/d relation are calculated and shown in Fig.10 as a function of w/d with parameter of thickness. It can be seen that the effect of thickness on the characteristic impedance is sensitive. Characteristic impedance for very thin strip (a few percent of the plate spacing) is calcurable by this method and shown in the same figure as a function of w/d.

\section{CONCLUSION}

New and systematic analysis method for stripline is presented, which is based on planar circuit and lateral equivalent network, and can give exact eigenmode for the stripline. How to calculate characteristic impedance of TEM mode is proposed and confirmed with success by applying the proposal to the practical problem. Also calculation error by this method is investigated by comparison with exact value given for $\mathrm{t}=0[\mathrm{~mm}]$. The agree- ment is good, which demonstrates the validity of this method. Also this method can give more exact characteristic impedance compared with that given by conventional fringing capacitance method whose accuracy is $1.2 \%$. Thus obtained more exact characteristic impedance is useful for exact analysis and design of stripline circuit.

\section{REFERENCES}

[1] R.E.Collin "Foundations for Microwave Engineering”McGraw-Hill,Inc pp170-171, TK7876-C645, 1992

[2] S.B.Cohn "Problems in Strip Transmission line" Volume MTT part2 pp119 126 March 1955

[3] Hsu.Jui-pang, T.Anada "Proposal of Surface-wave Planar Circuit, Formulation of its Planar Circuit equations and its Practical Application", 1986 IEEE MTT-S Digest, GG-4,pp797 800

[4] A.Hirota T.Hiraoka Hsu.jui-pang "Analysis of Propagation Eigenmode for Stripline based on Planar Circuit Equation and Lateral Equivalent Network” 2006 IEEE IMS Digest WEPA-03

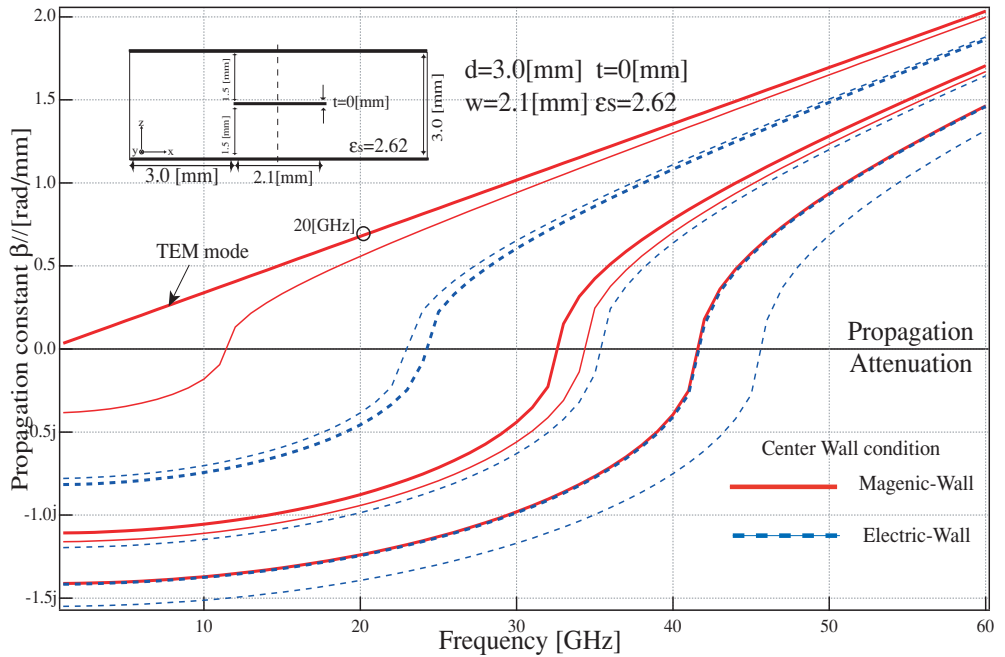

Fig.5 Frequency characteristics of propagation constants of TEM mode and higher modes for stripline of zero thickness $(\mathrm{t}=0.1[\mathrm{~mm}])$ where $70 \mathrm{TE} / \mathrm{TM}$ slab-modes are taken into consideration.
Ex component $\mathrm{w}=2.1[\mathrm{~m}$

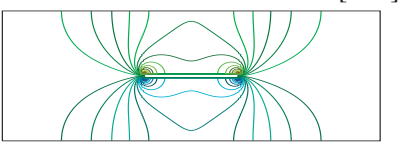

Hx component

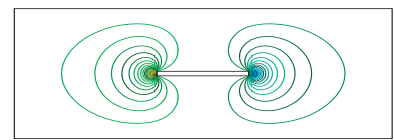

Ex component

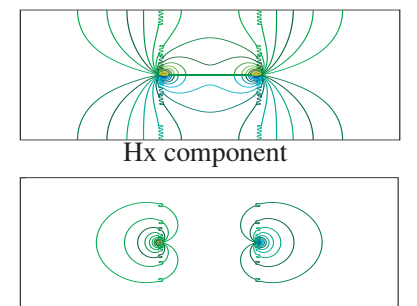

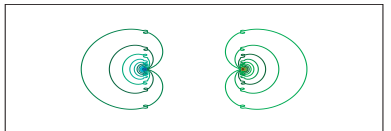

Hz component

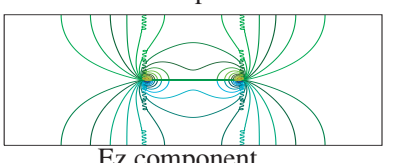

Ez component

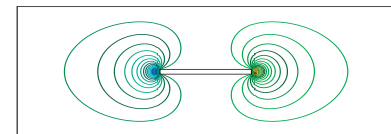

Hz component

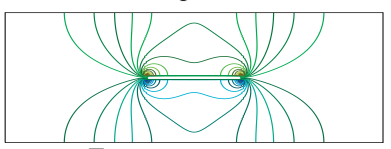

Ez component $\mathrm{W}=2.1[\mathrm{~mm}] \mathrm{t}=0.1[\mathrm{~mm}]$

Fig.6 Distribution of transverse electromagnetic field $\mathbf{E}_{\mathrm{t}}\left(\mathrm{E}_{\mathrm{x}}\right.$ and $\left.\mathrm{E}_{\mathrm{z}}\right)$ and $\mathbf{H}_{\mathrm{t}}$ $\left(\mathrm{H}_{\mathrm{X}}\right.$ and $\left.\mathrm{H}_{\mathrm{z}}\right)$ for various dimension of the strip where the calculation is carried out at 20 [Ghz] and $70 \mathrm{TE} / \mathrm{TM}$ slab-modes are taken into consideration.

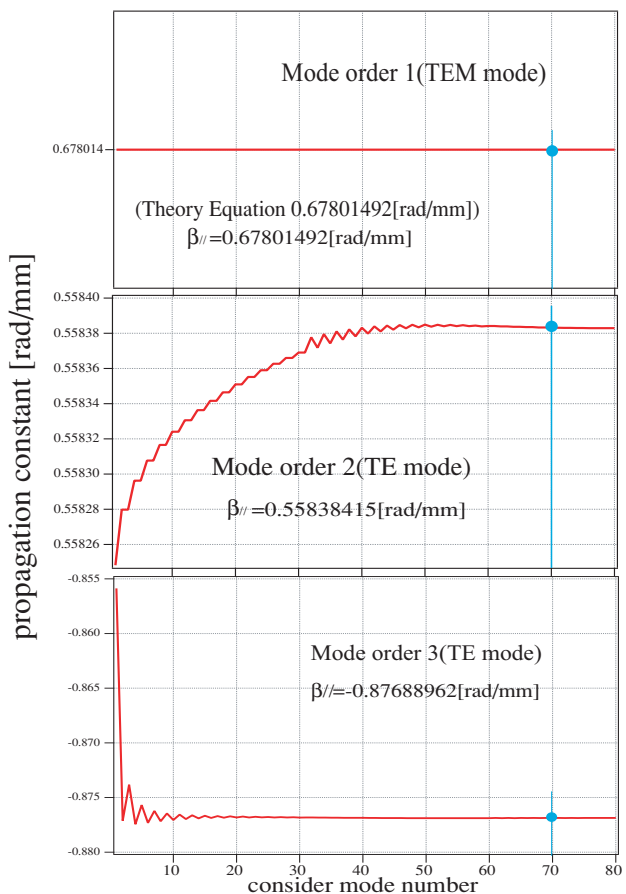

Fig.4 Convergence of propagation constant for stripline of $0.1[\mathrm{~mm}]$ thickness with number of height mode in \#3 region

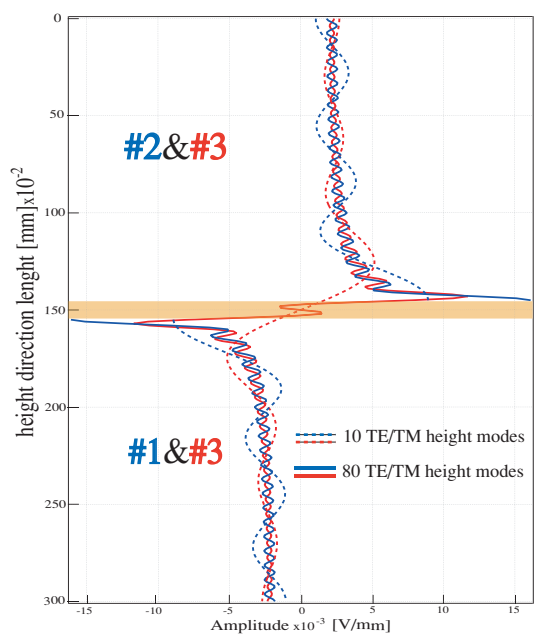

Fig.7 Continuity of the field component Hx at step discontinuitis \#2-\#3 and \#1-\#3 for $0.1[\mathrm{~mm}]$ thickness strip. 


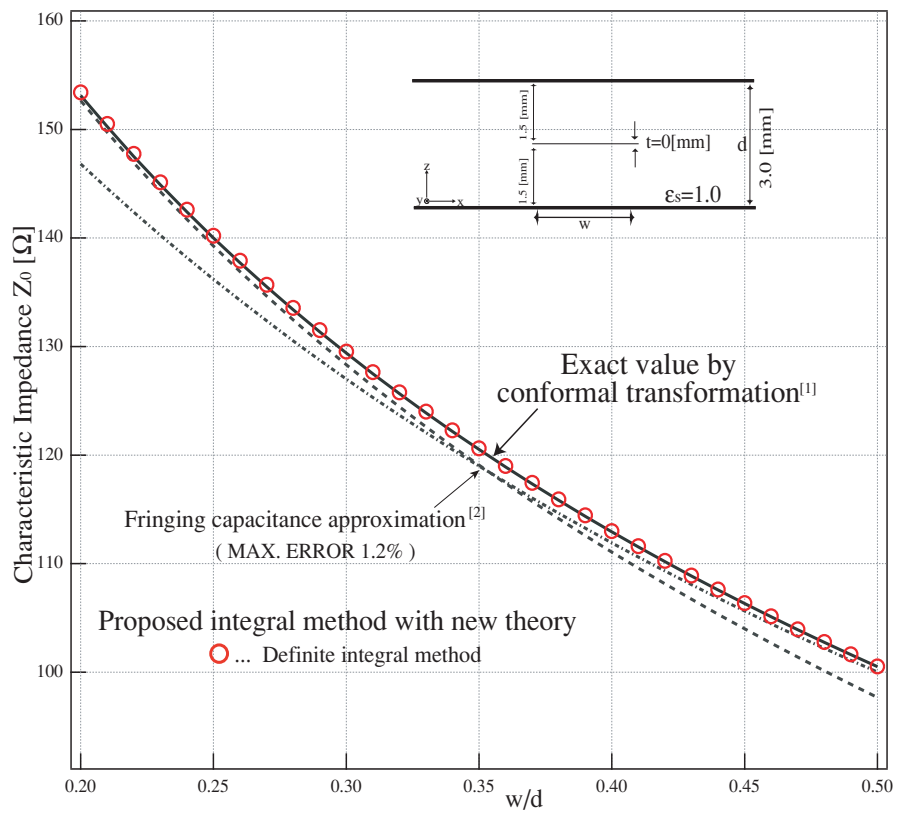

Fig. 8 Characteritic impedance vs w/d characteristics based on new theory/fringing capacitance approximation/exact conformal transformation for zero strip thickness

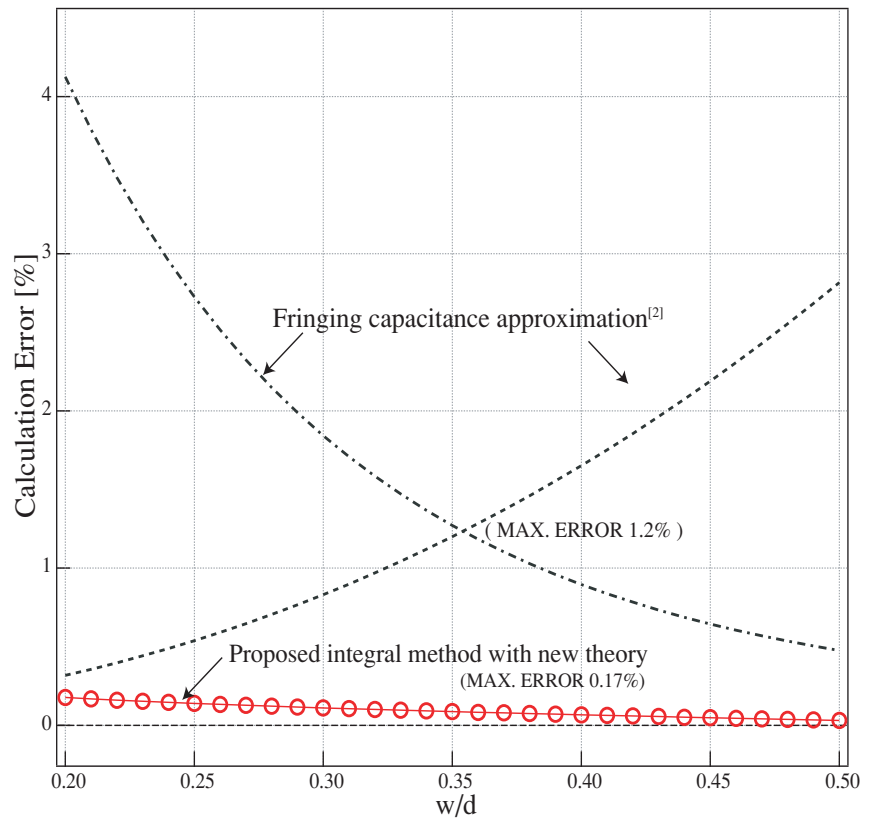

Fig. 9 Calculation error in \% vs w/d characteristics for new theory and fringing capacitance approximation for zero strip thickness based on exact conformal transformation

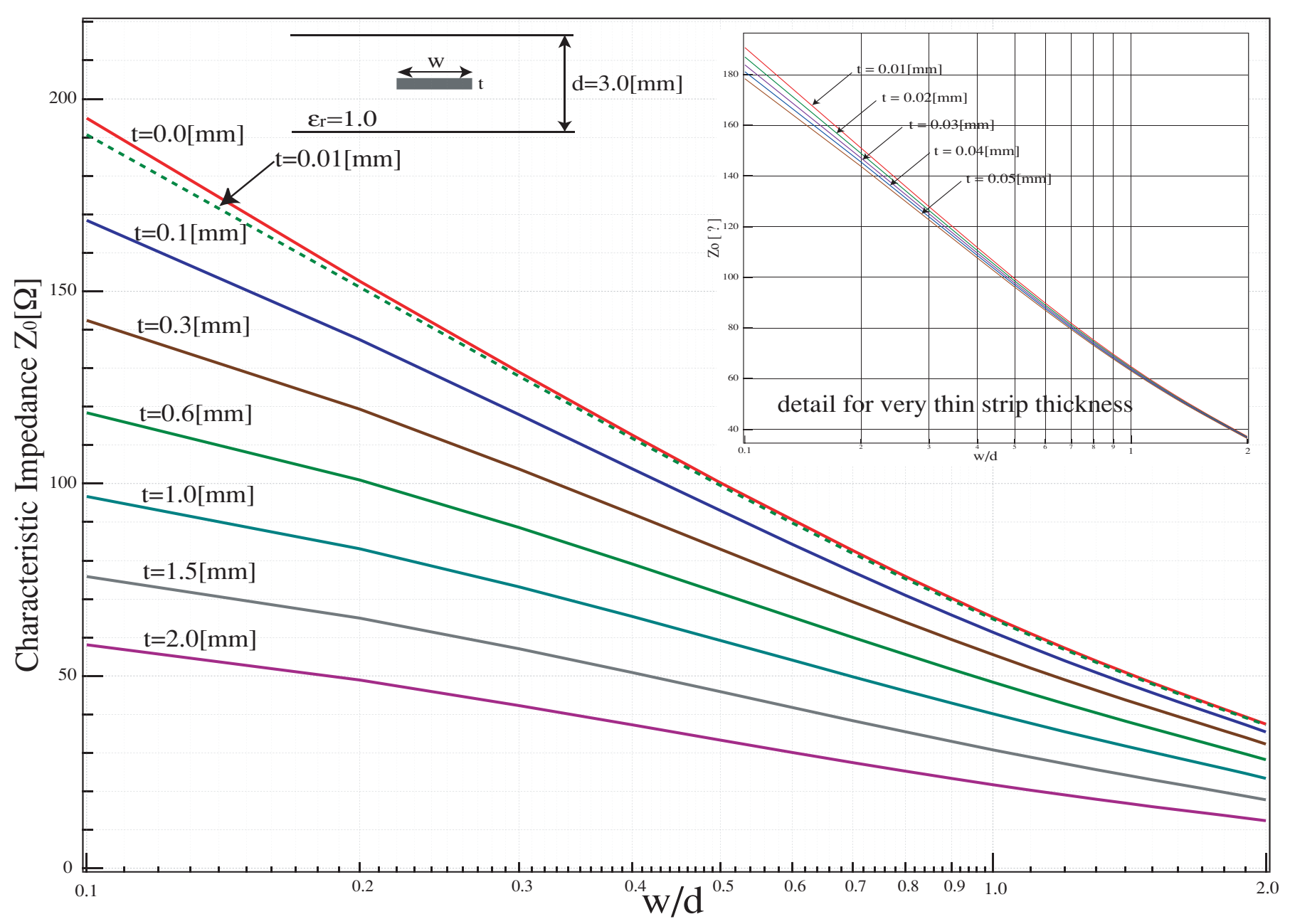

Fig.10 Characteristic impedance $\mathrm{Z}_{0} \mathrm{vs} w / \mathrm{d}$ characteristics with parameter of strip thickness $\mathrm{t}$ and their detailed characteristics for very thin strip thickness $t$ 